\section{Factores asociados a caídas en adultos mayores chilenos: evidencia de la Encuesta Nacional de Salud 2009-2010}

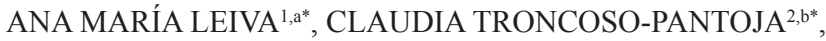

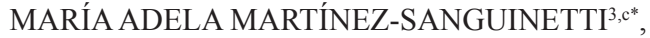 \\ FANNY PETERMANN-ROCHA ${ }^{4, d}$, FELIPE POBLETE-VALDERRAMA ${ }^{5, \mathrm{e}}$, \\ IGOR CIGARROA CUEVAS ${ }^{6, \mathrm{f}}$, CARLOS CELIS-MORALES ${ }^{7,8, \mathrm{~g}}$ \\ (En representación de todos los integrantes del grupo ELHOC)
}

\section{Factors associated with falls in older adults in chile. Analysis of the National Health Survey 2009-2010}

Background: A decrease in functional capacity due to ageing is one of the main risk factors for falls in older people. Aim: To investigate factors associated with falls in Chilean older adults. Material and Methods: We analyzed the self-reports of falls of 1,334 people aged $\geq 60$ years who answered the National Health Survey 2009-2010. Falls during the last 12 months were recorded. Lifestyle, socio-demographic and health status were analyzed. A Poisson regression with robust variance estimates was performed to identify factors associated with falls. Results: Falls during the preceding year were reported by $37 \%$ of respondents ( $95 \%$ confidence intervals (CI): 32-42]. Fall frequency was higher in women (Prevalence ratio (PR):1.30 [95\% CI:1.11; 1.53], $p<0.01)$ and those aged $\geq 75$ years (PR:1.29 [95\% CI:1.04; 1.61], $p=0.02)$. Hearing impairment (PR: 1.31 [95\% CI: 1.07; 1.61], $p<0.01$ ), impaired vision (PR:1.46 [95\% CI:1.20; 1.77], $p<0.01$ ), low self-reported wellbeing (PR: 1.41 [95\% CI: 1.03; 1.94], $p=0.03$ ) and disability (PR: 1.54 [95\% CI:1.32; 1.79], $p<0.01$ ) were associated with falls. However, multimorbidity (having $\geq 3$ diseases) was negatively associated with falls (PR: 0.79 [95\% CI: 0.63; 0.99], $p=0.04)$. Conclusions: Among older people, female sex, being aged $>75$ years and having disability, hearing or vision impairment are risk factors for falls.

(Rev Med Chile 2019; 147: 877-886)

Key words: Accidental Falls; Aged; Hearing Loss; Vision Impairment.
${ }^{1}$ Instituto de Anatomía, Histología y Patología, Facultad de Medicina, Universidad Austral de Chile. Valdivia, Chile.

${ }^{2}$ CIEDE-UCSC, Departamento de Salud Pública, Facultad de Medicina, Universidad Católica de la Santísima Concepción. Concepción, Chile. ${ }^{3}$ Instituto de Farmacia, Facultad de Ciencias, Universidad Austral de Chile.

Valdivia, Chile.

${ }^{4}$ Institute of Health and Wellbeing,

University of Glasgow. Glasgow, United Kingdom.

${ }^{5}$ Escuela de Kinesiología, Facultad de Salud, Universidad Santo Tomás, Sede Valdivia. Chile.

${ }^{6}$ Escuela de Kinesiología, Facultad de Salud, Universidad Santo Tomás, sede Los Ángeles, Chile.

${ }^{7} \mathrm{BHF}$ Glasgow Cardiovascular Research Centre, Institute of Cardiovascular and Medical Science, University of Glasgow. Glasgow, United Kingdom.

${ }^{8}$ Centro de Investigación en Fisiología del Ejercicio - CIFE, Universidad Mayor.

Santiago, Chile.

aProfesora de Biología y Química.

Magíster en Neurociencias y Salud Mental.

${ }^{\text {b}}$ Nutricionista. Magíster en Salud Pública. Magíster en Gerontología.

'Bioquímica. Magíster en Nutrición y Dietética.

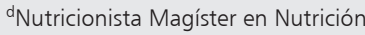
Humana.

eProfesor de Educación Física, Magíster en Educación en Salud y Bienestar Humano. ${ }^{f}$ Kinesiólogo. Doctor en Neurociencias. gProfesor de Educación Física. Doctor en Ciencias Cardiovasculares y Biomédicas. *AML, CTP y MAMS contribuyeron de igual manera a este manuscrito por ende son considerados como primer autor compartido.

Recibido el 6 de octubre de 2018 , aceptado el 8 de agosto de 2019

Correspondencia a:

Ana María Leiva Ordoñez Instituto de Anatomía, Histología y Patología, Facultad de Medicina, Universidad Austral de Chile. Valdivia, Chile. aleiva@uach.cl
L as caídas y sus consecuencias son una de las principales causas de morbilidad y mortalidad entre los adultos mayores. Cada año, se calcula que 646.000 personas mueren por caídas en todo el mundo, siendo los mayores de 65 años quienes sufren una mayor cantidad de caídas mor- tales $^{1-3}$. Las lesiones no fatales por su parte, causan lesiones graves, destacándose los traumatismos encefalocraneanos y fracturas de cadera, que implican hospitalización y dependencia ${ }^{4}$.

Según datos de la Organización Mundial de la Salud (OMS), entre 28 a 35\% de las personas 
mayores de 65 años experimentan alguna caída, porcentaje que aumenta progresivamente a medida que avanza la edad ${ }^{5}$. Entre las principales condicionantes que pueden actuar como predictores en las caídas de este grupo etario, son la disminución de la capacidad funcional, reducción en los niveles de fuerza muscular, flexibilidad, balance, niveles de actividad física (AF) así como también la conciencia del riesgo de caídas ${ }^{3}$.

En Chile, se estima que uno de cada tres adultos mayores presenta una o más caídas al año. La Encuesta de Salud, Bienestar y Envejecimiento (SABE) identificó una prevalencia anual de 35,3\% de caídas en adultos mayores ${ }^{6}$, resultando gran parte de estas caídas en fracturas ${ }^{7}$. Las caídas en los adultos mayores suelen ser de etiología multifactorial, representando un fenómeno traumático que constituye un problema de salud pública, ya que su impacto afecta a familias, comunidades y a la sociedad en varias dimensiones, al reducir el nivel de independencia, actividades sociales y calidad de vida; pudiendo desencadenar dependencia y postración ${ }^{8-10}$.

En Chile, los adultos mayores superan los 2,8 millones de personas, lo que equivale a $16,2 \%$ de los habitantes del país ${ }^{11}$, proyectándose para el 2025 que la población mayor de 60 años aumentará a $20 \%$ de la población nacional y la expectativa de vida llegará a 89 años en promedio. Es así que se habla de un proceso de "envejecimiento del envejecimiento"; es decir, las personas que se encuentran en el grupo de los adultos mayores están extendiendo su vida por sobre los 60 o 65 años ${ }^{12}$.

Frente a la realidad sociodemográfica que enfrenta Chile respecto al envejecimiento de su población, se requiere generar evidencia científica orientada a explorar los diferentes factores de riesgo asociados a caídas con el fin de desarrollar acciones con un enfoque preventivo. Por lo anterior, el objetivo de este estudio fue investigar los factores asociados a caídas en adultos mayores chilenos, tomando como base los resultados de la Encuesta Nacional de Salud 2009-2010.

\section{Materiales y Métodos}

\section{Diseño del estudio}

Este estudio incluyó a 1.334 participantes mayores de 60 años de la Encuesta Nacional de Salud de Chile (ENS 2009-2010). La ENS 2009-
2010 correspondió a un estudio de prevalencia realizado en hogares en una muestra nacional, probabilística, estratificada y multietápica de 5.412 personas mayores de 15 años con representatividad nacional, regional, y área urbana/rural. El protocolo de estudio fue aprobado por el Comité de Ética de la Escuela de Medicina de la Pontificia Universidad Católica de Chile. Todos los participantes firmaron un consentimiento informado para su participación ${ }^{13}$.

\section{Clasificación del adulto mayor}

Para este estudio se clasificó como adulto mayor a todas las personas $\geq 60$ años según las recomendaciones establecidas por la Organización de Naciones Unidas (ONU) para los países no desarrollados ${ }^{14}$. Para evaluar la asociación de la población según registro de caídas acorde avanza la edad, se subdividió a los adultos mayores en cinco grupos: 60-65 años, 66 a 70 años, 71 a 75 años, 76 a 80 años y $>80$ años.

\section{Variables Sociodemográficas y de estilos de vida}

Las variables sociodemográficas, estilos de vida y presencia de comorbilidades se obtuvieron mediante la aplicación de cuestionarios validados y descritos en la ENS 2009-2010 ${ }^{13}$. Las horas de sueño fueron autorreportadas clasificándose en 3 categorías ( $<7$ h/día, 7-9 h/día y $>9$ h/día). Para consumo de alcohol, sal, frutas y verduras se crearon variables binarias en base a las recomendaciones de consumo diario ${ }^{13}$. Los puntos de corte para consumo de alcohol fueron en base al puntaje AUDIT; consumo de riesgo $>8$ puntos AUDIT, para consumo de sal fue de $>8 \mathrm{~g} /$ día, para frutas y verduras $<5$ porciones/día. El consumo de sal se determinó mediante análisis de excreción de sodio en la orina a través de la fórmula de Tanaka ${ }^{15}$.

\section{Clasificación de nivel de actividad física}

El tiempo destinado a AF relacionada con el transporte y las actividades de intensidad moderada o vigorosa realizadas durante el tiempo libre y/o en el trabajo se determinó a través de la guía de análisis de GPAQ (Global Physical Activity Questionnaire v2 $)^{16}$. La AF total fue expresada en $\mathrm{MET} /$ hora/semana. Se consideró como punto de corte para inactividad física un gasto energético $<600 \mathrm{METs} / \mathrm{min} / \mathrm{semana}^{16}$. El tiempo sedente se determinó mediante el autorreporte del tiempo destinado a actividades que involucren estar 
sentado o reclinado durante el tiempo libre o de trabajo y fue medido en hora/día.

\section{Variables antropométricas}

El estado nutricional fue determinado a través del índice de masa corporal (IMC: $\mathrm{kg} / \mathrm{m}^{2}$ ) y clasificado según los puntos de corte específicos para adulto mayor (bajo peso: $<22,9 \mathrm{~kg} / \mathrm{m}^{2}$; normo peso: $23,0-27,9 \mathrm{~kg} / \mathrm{m}^{2}$; sobrepeso: $28,0-31,9 \mathrm{~kg} / \mathrm{m}^{2}$ y obesidad: $\left.\geq 32,0 \mathrm{~kg} / \mathrm{m}^{2}\right)^{17}$. La obesidad central fue definida midiendo el perímetro de cintura (PC), utilizando los puntos de corte sugeridos por la ENS 2009-2010 (PC $\geq 88 \mathrm{~cm}$ para mujeres y $\mathrm{PC} \geq 102$ cm para hombres $)^{13}$.

\section{Problemas auditivos}

Fueron autorreportados a partir de tres preguntas. A1: consideración de que escucha en forma normal por los dos oídos, A2: capacidad de seguir un programa de televisión a un volumen aceptable y A3: capacidad de seguir una conversación de tres o más personas ${ }^{13}$.

\section{Problemas visuales}

La calidad visual fue definida mediante autorreporte en base a la pregunta ${ }_{i}$ Usted piensa que su vista es? Con las siguientes opciones de respuesta: buena, regular y mala ${ }^{13}$.

\section{Sospecha de deterioro cognitivo}

Se aplicó la versión abreviada del Mini-Mental State Examination (MMSE), el que cuenta con 19 puntos a evaluar, considerando los resultados menores a 13 puntos como sospecha de deterioro cognitivo ${ }^{18}$.

\section{Discapacidad}

Para medir las dificultades funcionales de la vida cotidiana se utilizó el módulo de Health State de la WHS2003-OMS (18 preguntas) ${ }^{19}$, que explora 8 dominios de funcionalidad; instrumento propuesto por la Clasificación Internacional de Discapacidad, Funcionalidad y Salud, utilizándose la versión mexicana adaptada para la ENS 2009-2010 20 .

\section{Análisis estadístico}

Para los análisis se utilizó el módulo de análisis de muestras complejas del programa Stata/SE v15 y los resultados fueron estimados utilizando muestras expandidas según la ENS 2009-2010 ${ }^{13}$.
Los datos de caracterización de la población se presentan como promedio y porcentaje expandidos con sus respectivos $95 \%$ intervalos de confianza (95\% IC) para variables continuas y categóricas, respectivamente. Para determinar los principales factores asociados a caídas en los adultos mayores durante los últimos 12 meses, se aplicó el modelo de regresión de Poisson con estimación de varianza robusta y los resultados fueron expresado como Razón de Prevalencia y sus $95 \%$ IC. Debido a que la prevalencia de caídas supera el $10 \%$ en la población estudiada el modelo de regresión de Poisson presenta una mayor precisión en la estimación de la asociación que análisis más tradicionales como regresión logística ${ }^{21}$. El modelo de regresión de Poisson fue ajustado por edad, sexo, zona y región de residencia, nivel educacional, e IMC, a excepción de cuando estos fueron insertados dentro del modelo estadístico como variable de exposición principal. El nivel de significancia para las asociaciones fue definida con un valor $\mathrm{p}<0,05$.

\section{Resultados}

La prevalencia de caídas durante los últimos 12 meses en la población adulta mayor fue de $37,2 \%$ (95\% IC: 32,3; 42,2], siendo mayor en mujeres $(41,3 \%$ [95\% IC: 35,$4 ; 47,5])$ que en hombres (32,2\% [95\% IC: 24,6; 40,9]) (Figura 1). Al considerar la prevalencia de caídas por edad a los 60 años, fue de $26,2 \%$ y $35,4 \%$, aumentando a $49,0 \%$ y $53,7 \%$ a los 90 años en hombres y mujeres respectivamente (Figura 1).

La Tabla 1 presenta las características de la población estudiada, según reporte de caídas durante el último año. Se destaca que los adultos mayores que reportaron caídas, son en mayor porcentaje mujeres, viudos, jubilados, presentan discapacidad, problemas auditivos y de visión; tienen sobrepeso u obesidad y duermen $<7 \mathrm{~h} /$ día o $>9$ h/día.

Entre los factores sociodemográficos asociados a caídas en adultos mayores, se encuentra la edad, siendo los $>75$ años quienes presentaron una mayor asociación con caídas. En comparación a los hombres, las mujeres presentaron mayor asociación con sufrir caídas en los últimos 12 meses (Figura 2). Respecto a las condiciones de salud (Figura 3), las personas que reportaron tener $2 \mathrm{o}$ 


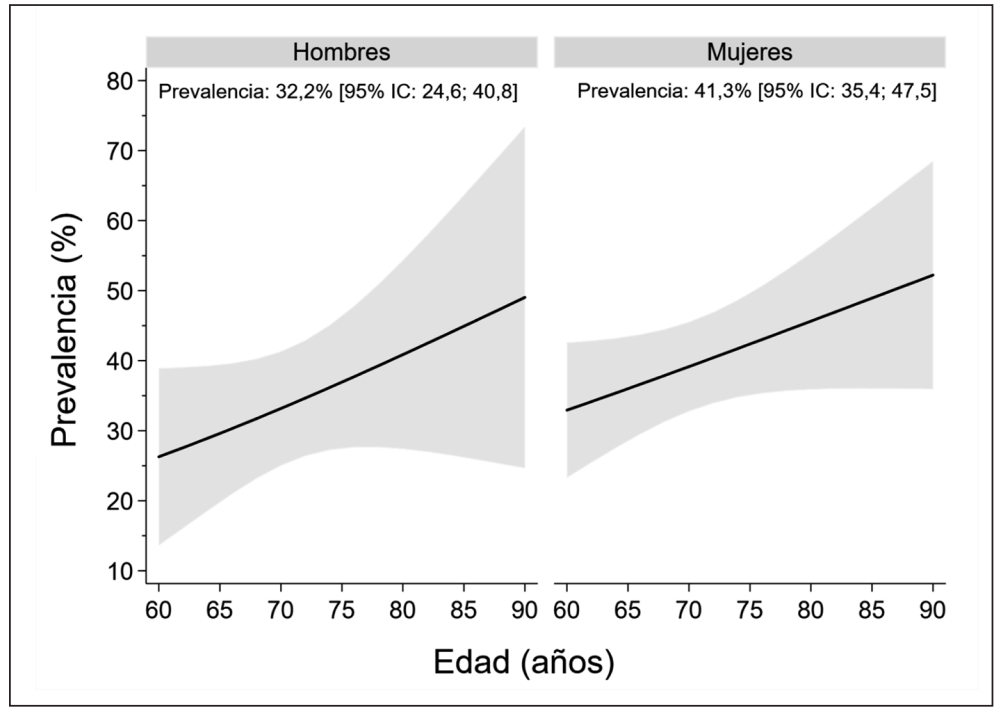

Figura 1. Prevalencia de caídas según edad y sexo. Datos presentados como prevalencias expandidas y sus respectivos $95 \%$ IC. La prevalencia total en hombres y mujeres se presenta en la parte superior de cada gráfico. La prevalencia de caídas en la población adulta mayor en general fue de $37,2 \%$ (95\% IC: 32,$3 ; 42,2$ ].

Tabla 1. Características de la población según registro de caídas en los últimos 12 meses

\begin{tabular}{|c|c|c|}
\hline & No reporta caídas & Sí reporta caídas \\
\hline \multicolumn{3}{|l|}{ Sociodemográficas } \\
\hline Muestra encuestada, (n) & 869 & 465 \\
\hline Muestra expandida, $(n)^{*}$ & 1.474 .053 & 872.039 \\
\hline Prevalencia expandida, (\%) & $62,8(57,7 ; 67,7)$ & $37,2(32,3 ; 42,2)$ \\
\hline Edad (años) & $68,6(67,8 ; 69,3)$ & $70,4(68,9 ; 71,8)$ \\
\hline $\begin{array}{l}\text { Sexo, }(\%) \\
\text { Hombres } \\
\text { Mujeres }\end{array}$ & $\begin{array}{l}67,7(59,1 ; 75,3) \\
58,7(52,4 ; 64,5)\end{array}$ & $\begin{array}{l}32,2(24,7 ; 40,9) \\
41,3(35,4 ; 47,5)\end{array}$ \\
\hline $\begin{array}{l}\text { Lugar de residencia, (\%) } \\
\text { Rural } \\
\text { Urbana }\end{array}$ & $\begin{array}{l}60,2(48,1 ; 71,0) \\
63,3(57,7 ; 68,7)\end{array}$ & $\begin{array}{l}39,8(28,9 ; 51,8) \\
36,6(31,3 ; 42,3)\end{array}$ \\
\hline $\begin{array}{l}\text { Estado civil, (\%) } \\
\text { Casado } \\
\text { Viudo } \\
\text { Soltero }\end{array}$ & $\begin{array}{l}63,4(58,5 ; 71,6) \\
56,9(47,4 ; 65,8) \\
58,1(41,3 ; 73,2)\end{array}$ & $\begin{array}{l}34,6(28,3 ; 41,5) \\
43,1(34,1 ; 52,5) \\
41,8(26,8 ; 58,6)\end{array}$ \\
\hline $\begin{array}{l}\text { Nivel educacional, (\%) } \\
\text { Básica }<8 \text { años } \\
\text { Media } 8-12 \text { años } \\
\text { Superior }>12 \text { años }\end{array}$ & $\begin{array}{l}59,9(53,6 ; 65,9) \\
68,4(59,1 ; 76,5) \\
60,0(42,4 ; 75,4)\end{array}$ & $\begin{array}{l}40,1(34,1 ; 46,3) \\
31,5(23,5 ; 40,9) \\
39,9(24,5 ; 57,5)\end{array}$ \\
\hline $\begin{array}{l}\text { Nivel de ingreso, }(\%) \\
\text { Bajo }(<\$ 250 \mathrm{mil}) \\
\text { Medio }(\$ 250-450 \mathrm{mil}) \\
\text { Alto }(>\$ 450 \mathrm{mil})\end{array}$ & $\begin{array}{l}64,6(58,5 ; 70,1) \\
61,9(52,1 ; 70,9) \\
67,2(46,8 ; 82,7)\end{array}$ & $\begin{array}{l}35,4(29,8 ; 41,2) \\
38,0(29,1 ; 47,8) \\
32,8(17,3 ; 53,1)\end{array}$ \\
\hline $\begin{array}{l}\text { Situación Laboral, (\%) } \\
\text { Trabajador } \\
\text { Jubilado }\end{array}$ & $\begin{array}{l}69,9(58,1 ; 79,4) \\
60,6(53,1 ; 67,4)\end{array}$ & $\begin{array}{l}30,1(20,5 ; 41,8) \\
39,4(32,5 ; 46,8)\end{array}$ \\
\hline Antropométricas & & \\
\hline Peso corporal (kg) & $71,9(70,4 ; 73,5)$ & $69,7(67,3 ; 72,0)$ \\
\hline Talla (m) & $1,58(1,57 ; 1,60)$ & $1,57(1,55 ; 1,58)$ \\
\hline $\operatorname{IMC}\left(\mathrm{kg} / \mathrm{m}^{2}\right)$ & $28,6(28,0 ; 29,2)$ & $28,4(27,5 ; 29,3)$ \\
\hline
\end{tabular}




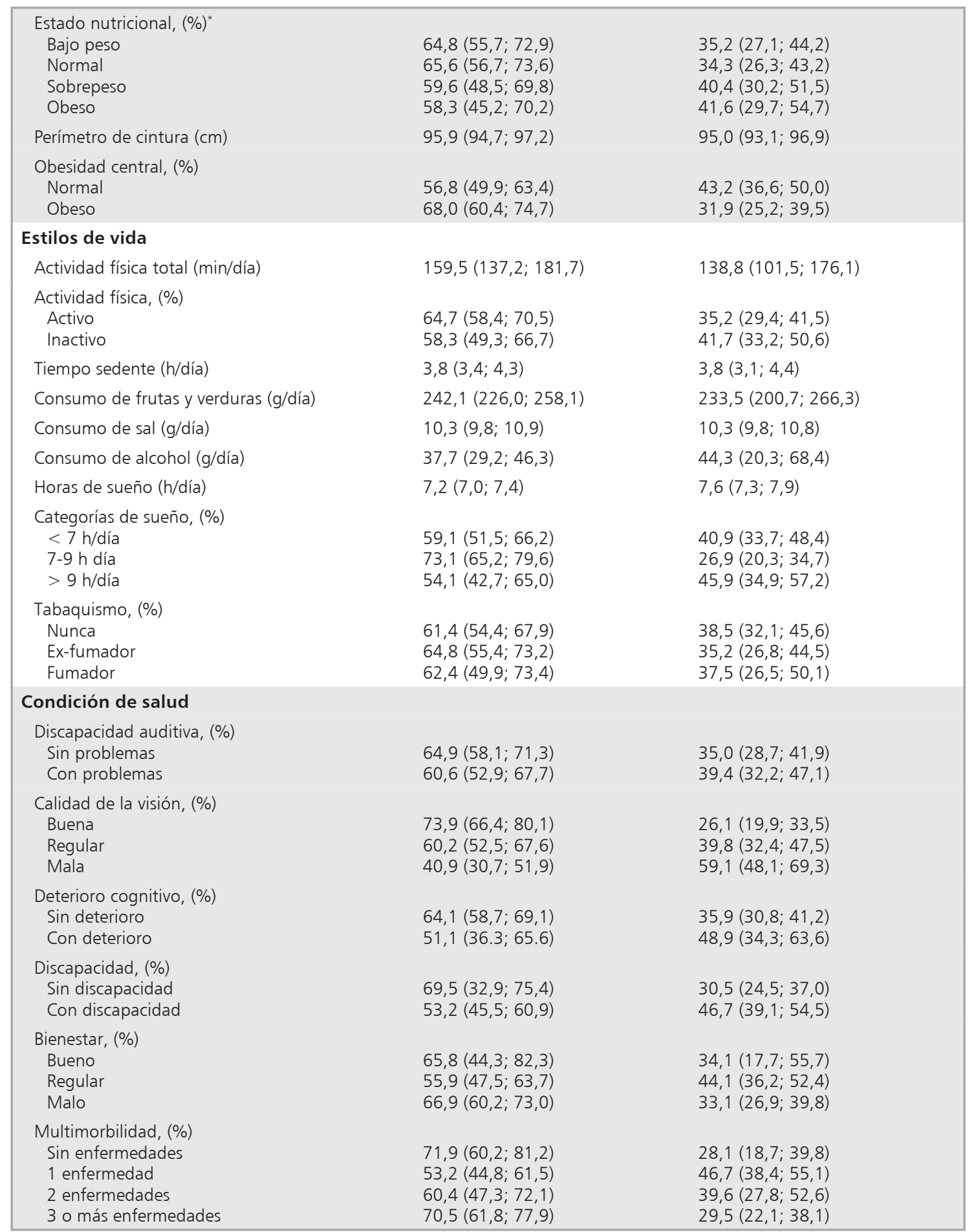

Datos presentados como promedio o prevalencia expandidas y su respectivo $95 \%$ intervalos de confianza para variables continuas y categorías, respectivamente. *Muestra expandida corresponde a la población nacional total estimada mediante los factores de expansión de la ENS 2009-2010. La muestra encuestada corresponde al total de personas incluidas en la ENS 2009-2010 pero sin aplicar ningún factor de expansión. 


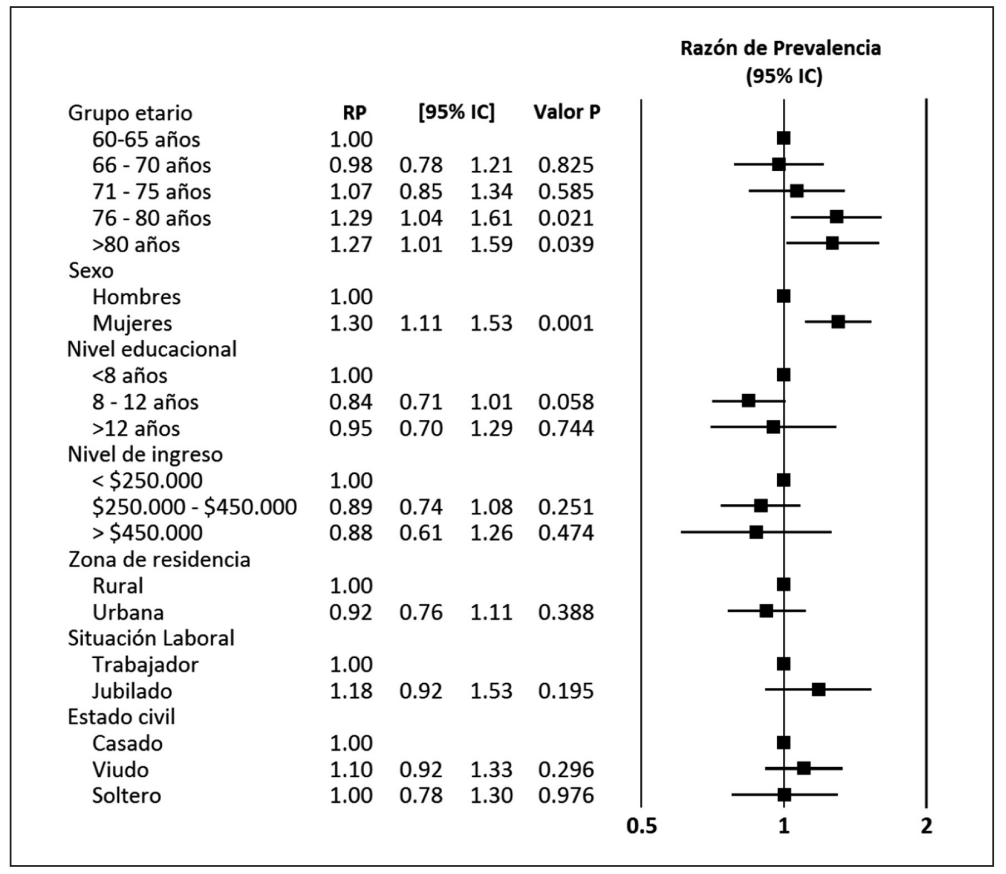

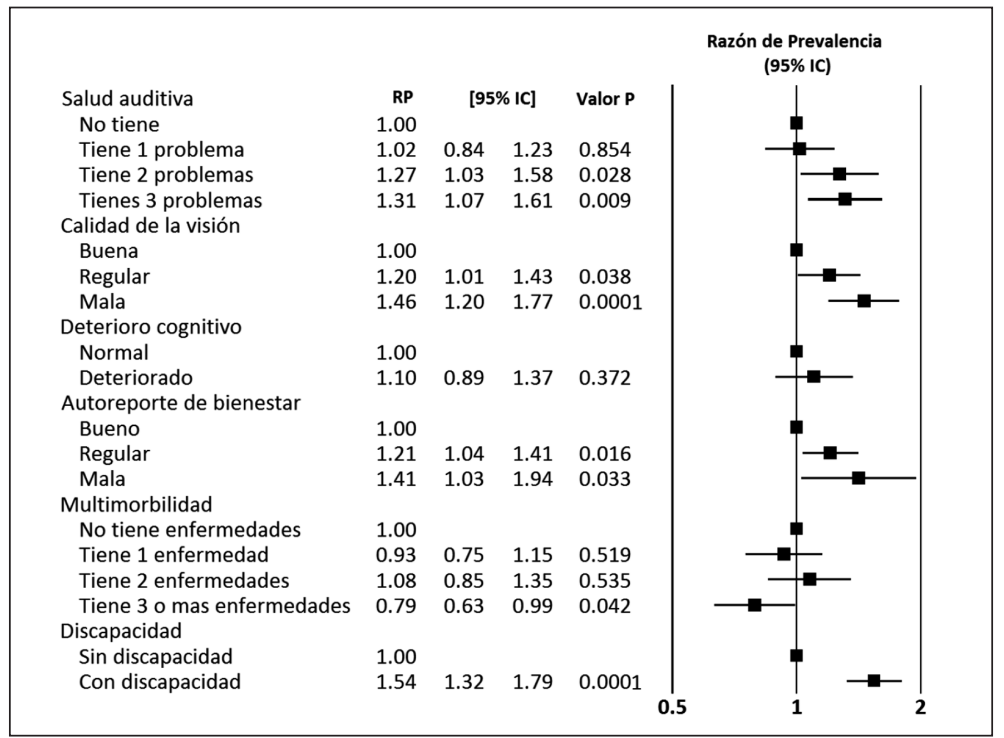

Figura 2. Factores sociodemográficos asociados a caídas en adulto mayor. Datos presentados como Razón de Prevalencia y sus respectivos 95\% IC (RP [95\% IC]. El análisis ajustado incluye las variables de edad, sexo, región, zona geográfica, nivel educacional e IMC, a excepción cuando estas variables fueron ocupadas como variable independiente o factor de riesgo en el modelo estadístico. Una razón de prevalencia $>1$ indica que hay una asociación positiva entre el factor de exposición y el haber sufrido una caída en los últimos 12 meses, mientras que una razón de prevalencia $<1$ indica una asociación negativa, es decir, una menor probabilidad de haber sufrido una caída.

Figura 3. Discapacidad y condiciones de salud asociadas a caídas en adultos mayores. Datos presentados como Razón de Prevalencia y sus respectivos $95 \%$ IC (RP $[95 \%$ IC]. El análisis ajustado incluye las variables de edad, sexo, región, zona geográfica, nivel educacional e IMC, a excepción cuando estas variables fueron ocupadas como variable independiente o factor de riesgo en el modelo estadístico. Una razón de prevalencia $>1$ indica que hay una asociación positiva entre el factor de exposición y el haber sufrido una caída en los últimos 12 meses, mientras que una razón de prevalencia $<1$ indica una asociación negativa, es decir, una menor probabilidad de haber sufrido una caída.
3 problemas auditivos, calidad de visión mala o regular, aquellos con discapacidad y quienes autorreportaron una percepción de bienestar regular o mala, presentaron una mayor asociación con caídas en el último año. Por el contrario, personas con multimorbilidad, es decir que tienen 3 o más enfermedades, presentaron una asociación negativa con la prevalencia de caídas. Los estilos de vida y estado nutricional no presentaron asociación con caídas sufridas en el último año (Figura 4). 


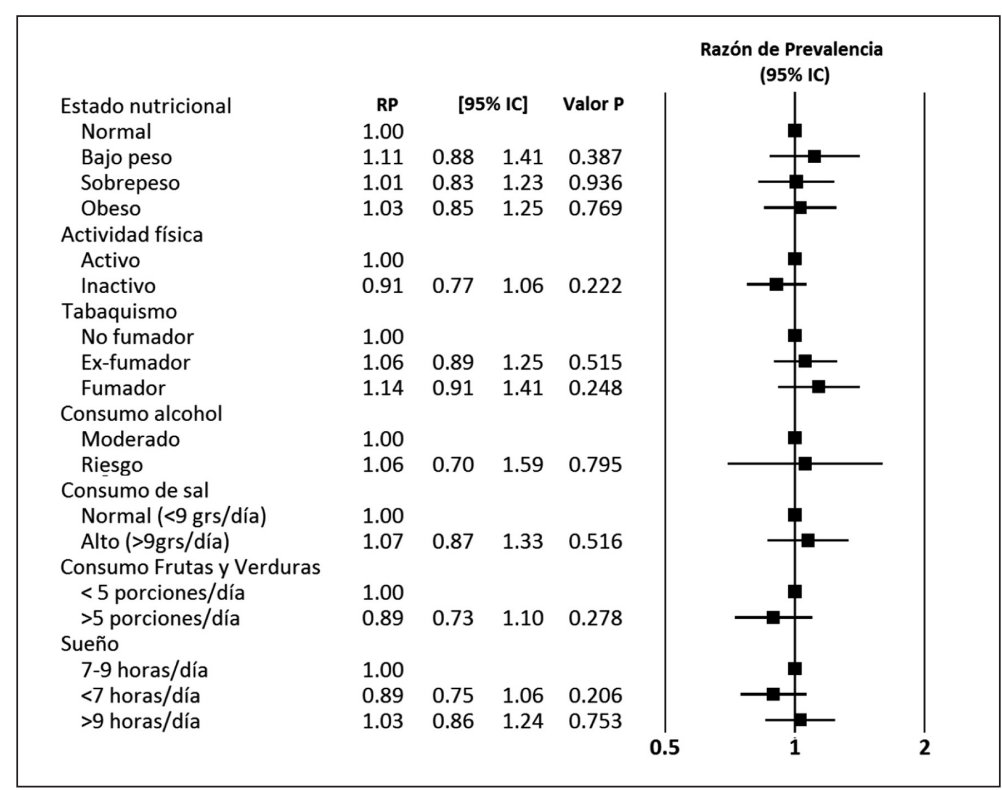

Figura 4. Estilos de vida asociados a caídas en adultos mayores. Datos presentados como Razón de Prevalencia y sus respectivos $95 \%$ IC (RP [95\% IC]. El análisis ajustado incluye las variables de edad, sexo, región, zona geográfica, nivel educacional e IMC, a excepción cuando estas variables fueron ocupadas como variable independiente o factor de riesgo en el modelo estadístico. Una razón de prevalencia $>1$ indica que hay una asociación positiva entre el factor de exposición y el haber sufrido una caída en los últimos 12 meses, mientras que una razón de prevalencia $<1$ indica una asociación negativa, es decir, una menor probabilidad de haber sufrido una caída.

\section{Discusión}

Treinta y siete coma dos porciento de los participantes adultos mayores declararon sufrir alguna caída en el último año, cifra superior al $11,6 \%$ reportado por la Encuesta de Calidad de Vida y Vejez UC - Caja los Andes realizada en Chile en el año $2017^{22}$, pero que está por debajo de prevalencias reportadas en otros países ${ }^{23,24}$.

La etiología de las caídas en los adultos mayores es multifactorial, destacándose factores intrínsecos como edad, género, sarcopenia, alteración del equilibrio y la marcha, polifarmacia e historial de caídas anteriores. Dentro de los factores extrínsecos o ambientales tenemos, la mala iluminación, calzado inapropiado y exposición al riesgo, como pisos resbalosos o en mal estado ${ }^{25-27}$.

Nuestros resultados identifican que la mayor probabilidad de presentar caídas en un año, se asocia con el sexo femenino, situación coincidente a lo reportado por diversas investigaciones realizadas a nivel global ${ }^{28-32}$ y nacional ${ }^{7,33}$. Manrique et al. encontraron que en las mujeres, ciertos factores biológicos que predeterminan una disminución de su masa muscular, las haría más propensas a presentar caídas, pero también estaría supeditado a una mayor presencia de problemas de salud y longevidad ${ }^{34}$.

Otro factor que condiciona las caídas es la edad del adulto mayor; encontrándose que a mayor edad existe más riesgo de caídas, situación similar se ha reportado en otros países latinoamericanos y europeos ${ }^{35-37}$.

Las alteraciones tanto visuales como auditivas, fueron otros factores claves asociados a caídas en la población en estudio. Resultados similares se han observado en estudios poblacionales en Latinoamérica, donde el déficit sensorial, tanto auditivo como visual ha representado un importante factor de riesgo de caídas en adultos mayores ${ }^{38,39}$.

En nuestro estudio, la presencia de multimorbilidad (coexistencia de 3 o más enfermedades) en los adultos mayores, presentó una asociación negativa con caídas, lo que no es coincidente con lo reportado en otros estudios, donde el riesgo de sufrir una caída ha sido asociado con la presencia de una o más enfermedades crónicas ${ }^{40}$. Esta diferencia podría explicarse en parte por el rol protector que implica el hecho de vivir acompañado y recibir el cuidado de sus cercanos, ya que en Chile $81,6 \%$ de los adultos mayores vive acompañado ${ }^{41}$.

Este estudio no identificó asociación entre deterioro cognitivo y caídas en adultos mayores; resultados que discrepan con estudios realizados en otros países, en los cuales el deterioro cognitivo ha sido identificado relevante para el riesgo de caídas ${ }^{42,43}$. La literatura reporta como causa de las caídas en estas personas, la desorientación vi- 
sual-espacial, lo que llevaría a una mala percepción de los riesgos ambientales ${ }^{44,45}$.

Otro aspecto importante a ser considerado en el manejo de las caídas del adulto mayor, es la práctica de actividad física, que influye directamente en su calidad de vida, evitando la sarcopenia y mejorando las funciones de equilibrio ${ }^{46,47}$. En nuestro estudio, los AM que reportaron caídas practicaban menos actividad física total, situación que podría explicarse con el miedo a caer $^{48}$.

Otro factor que puede afectar la calidad de vida del adulto mayor es el deterioro funcional. La pérdida de funcionalidad conlleva una discapacidad que representa un factor de riesgo importante para caídas; como lo evidenció nuestro estudio, donde los adultos mayores con discapacidad presentaron un riesgo aumentado de sufrir caídas, independiente de las variables confundidoras ${ }^{42,49}$.

Entre las principales limitantes de este estudio, está su diseño de corte transversal, que no permite establecer causalidad entre los factores asociados a caídas. Otras limitantes fue no considerar otros factores de riesgo importantes de caídas, como presencia de sarcopenia y uso de polifarmacia, que también podría ser un factor clave asociado a un mayor riesgo de caídas en este grupo etario.

\section{Conclusiones}

Ser mujer, tener una edad mayor de 75 años, presentar alguna discapacidad, tener problemas visuales o auditivos; son los factores de riesgo con mayor asociación de sufrir alguna caída en personas mayores en Chile

Las caídas son un grave problema de salud que las personas mayores inevitablemente enfrentan y que por lo mismo, deben ser consideradas a través de programas o intervenciones efectivas que reduzcan el riesgo a caer. En este sentido, este tipo de estudio de factores de riesgo modificable que se relacionan con las caídas en los adultos mayores, podrían ayudar a identificar poblaciones vulnerables y focalizar intervenciones orientadas a mitigar o prevenir las caídas a través del autocuidado.

\section{Referencias}

1. World Health Organization. What are the main risk factors for falls amongst older people and what are the most effective interventions to prevent these falls?
[Internet]. 2004. Available from: http://www.euro.who. int/_data/assets/pdf_file/0018/74700/E82552.pdf.

2. World Health Organization. Falls [Internet]. 2018. Available from: http://www.who.int/news-room/fact-sheets/ detail/falls.

3. Boneti N, Felix A, Pereira G, Barauce P. Does functional capacity, fall risk awareness and physical activity level predict falls in older adults in different age groups? Arch Gerontol Geriatr 2018; 77: 57-63. https://doi. org/10.1016/j.archger.2018.04.002.

4. Gutiérrez LM. Prevention of falls and their consequences. Eur Geriatr Med 2016; 7: 501-2.

5. World Health Organization. Global report on falls Prevention in older Age [Internet]. 2007. Available from: http://www.who.int/ageing/publications/Falls_prevention7March.pdf.

6. Organización Panamericana de la Salud. Salud, Bienestar y Envejecimiento en Santiago, Chile. SABE 2000.

7. Ministerio de Salud. Chile. Manual de Prevención de caídas en el adulto mayor. [Internet]. Available from: http://www.minsal.cl/portal/url/item/ab1f8c5957eb9d59e04001011e016ad7.pdf.

8. Worapanwisit T, Prabpai S, Rosenberg E. Correlates of Falls among Community-Dwelling Elderly in Thailand. J Aging Res 2018; Article ID 8546085 https://doi. org/10.1155/2018/8546085.

9. Bloch F, Boully C, Bourgoin G. Falls in the elderly, from the risk factors to prevention. Soins Gerontol 2015; 114: 10-2. DOI: 10.1016/j.sger.2015.05.003

10. Silva Z, Gómez A, Ferreira M. Epidemiology of falls in the elderly in Spain. A systematic review, 2007. Rev Esp Salud Pública 2008; 82 (1): 43-56. https://www.ncbi. nlm.nih.gov/pubmed/18398550.

11. Instituto Nacional de Estadística. Memoria CENSO 2017 [Internet]. Available from: http://www.censo2017. cl/memoria/descargas/memoria/libro_memoria_censal_2017_final.pdf.

12. Ministerio de Salud Chile. Programa Nacional de Salud de las Personas Adultas Mayores. 2014 [Internet]. Available from: http://www.minsal.cl/sites/default/files/ files/Borrador\%20documento\%20Programa $\% 20$ Nacional\%20de $\% 20$ Personas\%20Adultas\%20Mayores-\%20 04-03_14.pdf.

13. Ministerio de Salud Chile. Encuesta Nacional de Salud ENS Chile 2009-2010. [Internet]. Available from: http://web.minsal.cl/portal/url/item/bcb03d7bc28b64dfe040010165012d23.pdf.

14. Instituto para la Atención de los Adultos Mayores de la ciudad de México. Who is the older person? [Internet]. Available from: http://www.adultomayor.cdmx.gob.mx/ index.php/quien-es-el-adulto-mayor. 
15. Tanaka T, Okamura T, Miura K, Kadowaki T, Uesshima H, Nakagawa $\mathrm{H}$, et al. A simple method to estimate populational 24-h urinary sodium and potassium excretion using a casual urine specimen. J Hum Hypertens. 2002; 16 (2): 97-103. DOI: 10.1038/sj.jhh.1001307.

16. World Health Organization. Global Physical Activity Questionnaire: GPAQ version 2.0. World Health Organization. 2009. [Internet]. Available from: http:// www.who.int/ncds/surveillance/steps/resources/ GPAQ_Analysis_Guide.pdf.

17. OPS. Parte I: Módulos de Valoración clínica. Módulo 5: Valoración Nutricional del Adulto Mayor. Organización Panamericana de la Salud. 2003. Disponible en: http:// www.sld.cu/galerias/pdf/sitios/gericuba/modulo5.pdf.

18. Jiménez D, Lavados M, Rojas M, Henríquez C, Silva F, Guillón M. Performance of an abbreviated mini mental examination to detect dementia in older people. Rev Med Chile 2017; 145: 862-68.

19. World Health Survey 2002. Individual Questionnaire. World Health Organization, Evidence and Information for Policy. http://www.who.int/healthinfo/survey/whslongindividuala.pdf.

20. Secretaría de Salud. Encuesta Nacional de Salud y Nutrición, Cuestionario de adultos de 20 años y más. México, http://www.insp.mx/ensanut/cuestionarios/adultos.pdf.

21. Espelt A, Marí-Dell'Olmo M, Penelo E, Bosque-Prous M. Estimación de la Razón de Prevalencia con distintos modelos de Regresión: Ejemplo de un estudio internacional en investigación de las adicciones. Adicciones 2017; 29 (2): 105-12. http://www.adicciones.es/index. php/adicciones/article/view/823/776.

22. Fernández MB, Herrera MS. Chile y sus Mayores. 10 años de la Encuesta calidad de Vida en la Vejez UC Caja Los Andes. Programa Adulto mayor UC. Centro UC Estudios de Vejez y Envejecimiento. 2017.

23. Burton E, Lewin G, O'Connell, Hill K. Falls prevention in community care: 10 years on. Clin Interv Aging 2018; 13: 261-9. DOI https://doi.org/10.2147/CIA.S153687.

24. World Health Organization. What are the main risk factors for falls amongst older people and what are the most effective interventions to prevent these falls? 2004 [Internet]. Available from: http://iris.paho.org/xmlui/ bitstream/handle/123456789/741/9275316090.pdf?sequence $=1$.

25. World Health Organization. What are the main risk factors for falls amongst older people and what are the most effective interventions to prevent these falls? 2004 [Internet]. Available from: http://iris.paho.org/xmlui/ bitstream/handle/123456789/741/9275316090.pdf?sequence $=1$.

26. Ambrose A, Paul G, Hausdorff J. Risk factors for falls among older adults: A review of the literature. 2013; 75 (1): 51-61. https://doi.org/10.1016/j.maturitas.2013.02.009.

27. Sayer A, Syddall H, Martin H, Dennison E, Anderson F. Falls, Sarcopenia, and Growth in Early Life: Findings from the Hertfordshire Cohort Study. Am J Epidemiol 2006; 164 (7): 665-71. https://doi.org/10.1093/aje/ kwj255.

28. Yogi R, Sammy I, Paul J, Nunes P, Robertson P, Ramcharitar V. Falls in older people: comparing older and younger fallers in a developing country. Eur J Trauma Emerg Surg 2018; 44: 567-71. doi: 10.1007/s00068-0170818-2.

29. Araújo M, Nogueira N, Macial C, Malheiro F, Rodríguez M, López P. Record of the circumstances of falls in the community: perspective in the Iberian Peninsula. Rev. Latino-Am. Enfermagem 2018; 26: e2977http://dx.doi. org/10.1590/1518-8345.2373.2977.

30. Cruz P, Pérez A, Piloto A, Díaz D, Morales A, Reyes Y. Some causes related to falls in the home of the elderly. Rev Cubana Med Gen Integr 31 (1): 35-41. http://scielo. sld.cu/pdf/mgi/v31n1/mgi06115.pdf.

31. Huang S, Duong T, Ieong L, Quach T. Understanding Falls Risk and Impacts in Chinese American Older Patients at a Community Health Center. J Community Health. 2017; 42: 763-9. DOI 10.1007/s10900-017-0315-x.

32. Chirino D, Herrera G, Ferragut L, Osorio N. Risk factors associated with falls in the elderly at Hermanos Cruz teaching polyclinic. Rev Ciencias Médicas 2016; 20 (1): 0-0. http://scielo.sld.cu/scielo.php?script=sci_arttext\&pid=S1561-31942016000100011.

33. Leiva J, León J, Cortés J. Cane use and falls in chilean community dwelling older adults. Cienc. enferm. 2017; 23 (3): 11-21. http://dx.doi.org/10.4067/S071795532017000300011.

34. Manrique B, Salinas A, Moreno K, Téllez M. Functional dependency and falls in elderly living in poverty in Mexico. Salud Públ Méx 2011; 53 (1): 26-33. https:// www.scielosp.org/pdf/spm/2011.v53n1/26-33/es.

35. Silva J, Porras M, Guevara A, Canales R, Fabricio S, Partezani R. Risk of fall in the elderly to come to Day two Centers. Lima, Perú. Horiz. Med 2014; 14 (3): 12-8. http://www.scielo.org.pe/pdf/hm/v14n3/a03v14n3.pdf

36. Machado R, Bazán M, Izaguirre M. Main risk factors associated to falls in aged adults from Guanabo health area. MEDISAN 2014; 18 (2): 158-64. http://scielo.sld. cu/pdf/san/v18n2/san03214.pdf.

37. Gale C, Westbury L, Cooper C, Dennison E. Risk factors for incident falls in older men and women: the English longitudinal study of ageing. BMC Geriatrics 2018; 18: 117. https://doi.org/10.1186/s12877-018-0806-3. 
38. Villalobos-Cambronero X, Kulzer-Homann K, Fernández-Rojas X. Relación entre la funcionalidad y caídas en la población adulta mayor del proyecto Creles "Costa Rica, Estudio Longitudinal de Envejecimiento Saludable”. Rev Hisp Cienc Salud 2016; 2 (4): 294301.

39. Canales A. Factores Asociados al Riesgo de Caídas en Adultos Mayores de Consultorio Externo del Hospital Goyeneche, Arequipa. ALICIA, 2018.

40. Silva Fhon J, Marques S, Kusumota L, Carmo M. Enfermedades crónicas auto-reportadas asociadas a caídas em adultos mayores brasileiros. Rev Fac Cien Med (Quito) 2014; 39(2): 18-24.

41. Ministerio de Desarrollo Social. Informe de Desarrollo Social 2018. Gobierno de Chile. http://www.ministeriodesarrollosocial.gob.cl/storage/docs/Informe_de_Desarrollo_Social_2018.pdf.

42. Albornos L. Guía de buenas prácticas prevención de caídas y lesiones derivadas (4a Edición). Intervenciones efectivas para identificar adultos en riesgo de caídas. Ene 2018; 12 (1): 797. http://scielo.isciii.es/pdf/ene/ v12n1/1988-348X-ene-12-01-797.pdf.

43. Laguna J, Carrascosa R, Zafra F, Carrascosa M, Luque F, Alejo J, et al. Effectiveness of interventions for prevention falls in the elderly: systematic review. Gerokomos 2010; 21 (3): 97-107. http://scielo.isciii.es/pdf/geroko/ v21n3/revision1.pdf.

44. De Azevedo A, Oliveira A, Partezani R, Silva M, De Almeida J, Ragel L. Evaluación del riesgo de caídas en adultos mayores que viven en el domicilio. Rev. Latino-Am. Enfermagem 2017; 25: e2754. DOI: 10.1590/1518-8345.0671.2754.

45. Booth V, Harwood R, Hood V, Bramley T, Hancox J Van Der Wardt, et al. Promoting activity, independence and stability in early dementia and mild cognitive impairment (PrAISED): development of an intervention for people with mild cognitive impairment and dementia. Clin Rehabil 20118; 32 (7): 855-64.

46. Cruz P, Pérez A, Piloto A, Díaz D, Morales A, Reyes Y. Some causes related to falls in the home of the elderly. Rev Cubana Med Gen Integr 2015; 31 (1): 35-41. http:// scielo.sld.cu/pdf/mgi/v31n1/mgi06115.pdf.

47. Castro L, Galvis C. Effects of physical activity on cognitive impairment and dementia. Rev Cub Salud Pública 2018; 44 (3): e979. https://www.scielosp.org/pdf/ rcsp/2018.v44n3/e979/es.

48. Phelan EA, Mahoney J, Voit JC, Stevens JA. Assessment and Management of Fall Risk in Primary Care Settings. Med Clin N Am 2015; 99 (2): 281-93.

49. Molés P, Lavedán A. Prevalence and associated factors of fear of falling in older adults in Castellón de la Plana. Gerokomos 2017; 28 (4): 78-83. 